\title{
Reduced analgesic effect of acupuncture-like TENS but not conventional TENS in opioid treated patients
}

\author{
Guillaume Léonard ${ }^{1}$, Christian Coutier ${ }^{1}$, Serge Marchand ${ }^{1}$
}

${ }^{1}$ Université de Sherbrooke, Faculté de médecine, Sherbrooke, Québec, Canada, J1H 5N4.

Pages: 24

Tables: 4

Figures: 3

Corresponding author: $\quad$ Serge Marchand, Ph.D.

Université de Sherbrooke

Faculté de médecine, neurochirurgie

$3001,12 \mathrm{e}$ avenue nord

Sherbrooke (Québec) Canada J1H 5N4 


\begin{abstract}
Evidence from recent animal studies indicates that the analgesic effect of low frequency TENS is reduced in opioid tolerant animals. The aim of the present study was to compare the analgesic effect of conventional (high frequency) and acupuncture-like (low frequency) TENS between a group of opioid treated patients and a group of opioid-naive patients in order to determine if this cross-tolerance effect is also present in humans. Twenty-three chronic pain patients (11 who took opioids and 12 who did not) participated in the study. Participants were assigned in a randomized cross-over design to receive alternately conventional and acupuncture-like TENS. There was a significant reduction in pain during and after conventional TENS when compared to baseline for both the opioid and non-opioid group $(\mathrm{p}<.01)$. For acupuncture-like TENS however, the analgesic effect of TENS was only observed in the non-opioid group $(\mathrm{p}<.01)$, with opioid treated patients showing no change in pain scores during and after TENS when compared to baseline $(p>.09)$. The reduced analgesic effect of acupuncture-like TENS in opioid treated patients is coherent with previous animal studies and suggests that conventional TENS should be preferred in patients taking opioids on a regular basis.
\end{abstract}

Perspective: This study shows that patients taking opioids on a regular basis are less susceptible to benefit from acupuncture-like TENS. This phenomenon is probably attributable to the fact that the analgesia induced by acupuncture-like TENS and opioids are mediated by the same receptors (i.e. $\mu$ opioid receptors).

Keywords: Transcutaneous electrical nerve stimulation (TENS), opioids, rehabilitation 


\section{Introduction}

Transcutaneous electrical nerve stimulation (TENS) is a non-invasive modality commonly used in rehabilitation for pain relief. ${ }^{30}$ TENS applications are generally described according to the output characteristics of the device as either high frequency, low intensity (conventional TENS or CT) or low frequency, high intensity (acupuncturelike TENS or AT). ${ }^{10,14}$ The high frequency, low intensity stimulations employed by CT recruit $A \beta$ fibers which, according to the gate control theory of pain, inhibit the transmission of nociceptive information in the dorsal horn of the spinal cord. ${ }^{14,27,40}$ Alternately, the low frequency, high intensity stimulations used by AT activate $\mathrm{A} \delta$ and C fibers, producing counter-irritation analgesia via the recruitment of descending inhibition mechanisms. $^{43}$

There is growing evidence to suggest that the analgesic effect of TENS is associated with the release of endogenous opioids. ${ }^{8,12,20,38}$ Interestingly, the type of opioid receptor subserving TENS analgesia would depend on the stimulation parameters used, with high frequency stimulations producing analgesia through $\delta$ opioid receptors and low frequency stimulations producing analgesia through $\mu$ opioid receptors. ${ }^{20,23,38}$ The implication of opioid receptors in TENS analgesia could help to explain why the analgesic effect of TENS is sometimes found to decrease after repeated applications. ${ }^{13}$ This tolerance phenomenon (which is well documented for opioids) ${ }^{36}$ was described by Chandran \& Sluka ${ }^{7}$ who also noticed that animals rendered tolerant to high and low frequency TENS were tolerant to $\delta$ and $\mu$ opioid agonists, respectively. Moreover, the same group have shown that animals that were made tolerant to morphine (a $\mu$ opioid receptor agonist) were also tolerant to low frequency TENS. ${ }^{39}$ In contrast, the analgesic 
effect of high frequency TENS (which is believed to act on $\delta$ opioid receptors) was preserved in morphine-tolerant rats, suggesting that this cross-tolerance phenomenon is receptor specific.

If the same results are found in human, the studies of Sluka and colleagues ${ }^{7,39}$ can have several important implications for the clinician. First, and because the vast majority of opioids target $\mu$ opioid receptors, ${ }^{17}$ they would suggest that AT tolerant patients would retain less benefits from opioid analgesics than patients who are not tolerant to AT. Second, they would propose that the analgesic effect of AT would be considerably decreased in opioid-tolerant patients. In 1980, Solomon et al ${ }^{41}$ reported that the analgesic effect of TENS for post-operative pain was reduced in patients who had used narcotics prior to surgery compared to patients who had not used narcotics before surgery. These results suggests that the "cross-tolerance" phenomenon observed between TENS and opioids in rodents is also present in humans. ${ }^{39}$ Nevertheless, two important limitations prevent us from making any clear assertions. First, the results of Solomon et al. were based on posteriori analyses and should therefore be considered exploratory until confirmed by other studies. Second, and most importantly, the stimulation parameters for TENS were not specified by Solomon et al., making it impossible to determine if the "cross-tolerance" effect observed by the authors were between opioid analgesics and AT or between opioid analgesics and CT. Thus, the purpose of this study was to compare the analgesic efficacy of CT and AT between a group of patients who took opioids on a regular basis and a group of patient who did not use opioids for more than 6 months. Based on the work of Sluka et al., ${ }^{39}$ we 
hypothesized that the analgesic efficacy of AT (but not CT) would be decreased in patients who took opioids on a regular basis.

\section{Materials and Methods}

Participants

Twenty-three chronic pain patients ( 11 who took opioids on a daily basis for more than 4 months and 12 who did not use opioids for more than 6 months) participated in the study. Four patients from the non-opioid group previously took opioids for their pain condition but the medication was stopped more than 6 months before testing (mean time since medication was stopped $\pm \mathrm{SD}=25.8 \pm 23.9$ months). Participants were recruited through ads posted in local newspapers, physiotherapy clinics and in the Sherbrooke University Hospital's Pain Clinic. All participants had localized pain of diverse origin (e.g. spinal disc herniation, osteoarthritis, chondromalacia) for more than 6 months. For security reasons, patients with demand-type cardiac pacemakers and pregnant women were excluded. Every participant was asked to refrain from using short term analgesics two hours before testing and from taking caffeine and smoking cigarettes six hours before testing. Participants' characteristics are presented in Tables 1 and 2.

The experiment took place at the Clinical Research Centre of the Sherbrooke University Hospital, in Sherbrooke, Quebec, Canada. The Research Ethics Board for Human Subjects of the Sherbrooke University Hospital approved the study's procedures and each participant provided informed consent before participation. 
Experimental design and TENS stimulation protocol

Participants were assigned in a randomized cross-over design to receive, alternately, CT and AT. The order of presentation of the two types of TENS stimulations was determined using a random number table. This resulted in 11 participants receiving CT before AT, and 12 participants receiving AT before CT. Every participant was submitted to the two types of stimulation (approx. 1 week interval). At each visit, a thorough examination was made by the experimenter to determine the precise location of the pain. The painful area's margins were marked carefully with a pen in order to ensure optimal electrode placement. Participants were placed in a comfortable position, generally lying on their stomach (ventral decubitus) on a mobilization table. Pillows were given to ensure proper positioning.

TENS stimulations were delivered using a pair of rubber silicone electrodes connected to a digital Eclipse Plus apparatus (Empi, St-Paul, Minnesota). The electrodes were placed over the painful area identified previously. Electrodes' position was reassessed with the TENS stimulator turned $\mathrm{ON}$ in order to be certain that induced paresthesias entirely covered the painful region. In cases where stimulations did not properly cover the painful region, the stimulator was turned OFF and the electrodes were repositioned. For $\mathrm{CT}$, the frequency was set at $100 \mathrm{~Hz}$, the pulse duration at $60 \mu \mathrm{s}$, and the intensity was adjusted to produce strong and comfortable (innocuous) tingling sensations. For the AT, the frequency was set at $3 \mathrm{~Hz}$, the pulse duration at $250 \mu$ s and the intensity was adjusted to produce strong and painful sensations (pain tolerance threshold). For both CT and AT, the stimulation was applied for 25 minutes and the 
intensity was occasionally raised (based on the participant's sensation) to account for nerve accommodation and to maintain the same level of sensation. ${ }^{34,42}$

Pain intensity and unpleasantness was evaluated using two separate numerical rating scales (NRS) (intensity $0=$ no pain, $100=$ most intense pain imaginable; unpleasantness $0=$ not unpleasant, $100=$ most unpleasant pain imaginable). Pain intensity (sensory-discriminative component) and unpleasantness (motivational-affective component) are two distinct components of pain which are associated with distinct anatomical regions of the pain matrix. ${ }^{2}$ The distinctions between pain intensity and pain unpleasantness was explained successfully to the participants by using the analogy of Price and colleagues. ${ }^{32}$ Participants were asked to evaluate the intensity and unpleasantness of their clinical pain at three occasions: (1) before TENS application, (2) during TENS application (i.e. after 15 minutes of stimulation) and (3) immediately after TENS application. The Patient Global Impression of Change (PGIC) scale was also used after each TENS application to document participants' overall evaluation of their treatment. ${ }^{11,18}$

\section{Data analysis}

Because of the relatively small number of subjects included in this study, and since visual inspection of the histograms and normality tests (Shapiro-Wilk) did not allow us to assume that the data were normally distributed, non-parametric tests were used. Differences were considered to be significant if $\mathrm{p}<0.05$ was obtained. All tests were performed using SPSS (version 13.0 for Windows ${ }^{\circledR}$, Chicago, IL, USA). 


\section{Results}

Pain intensity and unpleasantness scores obtained prior to CT and AT applications showed that every participant experienced pain before TENS application (all baseline NRS scores $\geq 10$ ). Furthermore, Mann-Whitney tests conducted to test for group differences in baseline NRS scores revealed no significant group effect (all p-values $>$ .19), indicating that the opioid and non-opioid group had comparable pain intensity and unpleasantness scores before TENS application.

TENS stimulation threshold and intensities are shown in Table 3. As it can be seen from the table, stimulation threshold and intensities for AT were similar between the opioid and non-opioid groups $(\mathrm{p} \geq .40)$. For $\mathrm{CT}$, however, stimulation threshold and stimulation intensities were consistently higher in the opioid group $(\mathrm{p} \leq .03)$.

\section{Pain intensity}

Mean pain intensity scores obtained for each group before, during, and after CT and AT applications are shown in Figure 1A and 1B, respectively. There was a significant decrease in pain intensity during and after CT for both the opioid and nonopioid group. For AT however, only the non-opioid group showed a reduction in pain intensity. These differential effects were confirmed by the non-parametric analyses. First, Friedman's Test for CT revealed that there was a significant change in pain intensity across the different time measures for both the opioid and non-opioid groups (all p-values $<.001)$. Post-hoc Wilcoxon Signed-Rank Tests showed that there was a significant reduction in pain intensity during and after CT in both the opioid and nonopioid groups when compared to baseline (all p-values $<.01$ ). For the AT, Friedman's 
Test revealed a significant change in pain intensity across the different time measures only for the non-opioid group ( $\mathrm{p}<.001)$, while there was no change for the opioid group $(\mathrm{p}=.09)$. Post-hoc tests for the non-opioid group showed that there was a significant reduction in pain intensity during and after AT when compared with baseline (all pvalues $<.01)$.

Between-group analyses using Mann-Whitney Tests revealed significant differences between the opioid and non-opioid groups for pain intensity during $(\mathrm{p}<.01)$ and after $(\mathrm{p}<.05)$ AT but not before AT ( $p>18)$, suggesting that the variable "group" only had and effect on pain intensity during and after AT stimulations. There was no difference between the opioid and non-opioid groups for pain intensity before $(\mathrm{p}=.24)$, during $(\mathrm{p}=.74)$, and after $(\mathrm{p}=.29)$ CT .

\section{Pain unpleasantness}

Analyses performed for pain unpleasantness revealed the same pattern of results. That is, Friedman' Tests for CT showed a significant change in pain unpleasantness across the different times measures for both the opioid and non-opioid groups (all pvalues $<.001$ ). Post-hoc Wilcoxon Tests showed that there was a significant reduction in pain unpleasantness during and after $\mathrm{CT}$ in both the opioid (all p-values $<.05$ ) and non opioid group (all p-values $<.01$ ) when compared with baseline (see Figure 2A). For AT, Friedman's Tests revealed a significant change in pain unpleasantness across the different time measures only for the non-opioid group $(\mathrm{p}<.001)$, while there was no change for the opioid group ( $\mathrm{p}=.36$ ). Post-hoc Wilcoxon Tests for the non-opioid group showed that 
there was a significant reduction in pain unpleasantness during and after AT when compared with baseline (all p-values $<.01$; see Figure $2 \mathrm{~B}$ ).

Between-group analyses using Mann-Whitney Tests revealed significant differences between the opioid and non-opioid groups for pain unpleasantness during $(\mathrm{p}<.01)$ and after $(\mathrm{p}<.05)$ AT but not before AT $(\mathrm{p}>.18)$, suggesting again that the variable "group" only had and effect on pain unpleasantness during and after AT stimulations. There was no difference between the opioid and non-opioid group for pain unpleasantness before $(p=.46)$, during $(p=.38)$, and after $(p=.13)$ CT.

\section{Percentages of pain reductions}

In order to directly compare the analgesic effect of TENS stimulations, percentages of pain reductions obtained during CT and AT were calculated for each group $[$ pain reduction $=($ pain before TENS - pain during TENS $) /$ pain before TENS * 100]. These percentages are illustrated in Figure 3. As it can be seen from the figure, the analgesic effect of AT was substantially reduced in opioid patients. The reduced analgesic effect of AT in the opioid group was confirmed by the Wilcoxon Signed-Rank Test and by the Mann-Whitney Test which showed (i) that there was a significant difference in analgesia between CT and AT for the opioid group $(\mathrm{p}<.01)$ and (ii) that there was a significant difference in AT analgesia between the opioid and non-opioid groups $(\mathrm{p}<.01)$. Comparisons between CT and AT analgesia for the non-opioid group and comparisons of CT analgesia between the opioid and non-opioid groups revealed no significant differences (all p-values >.10). 


\section{PGIC scores}

PGIC scores for the CT and AT are represented in Table 4. As it can be seen from the table, the majority of patients from both the opioid and non-opioid groups reported significant improvements following CT stimulations. For AT however, less than half of the participants from the opioid group reported improvements following TENS, while the vast majority of the non-opioid group continued to benefit from the TENS. These between-group differences for the AT stimulations were confirmed by the Fisher's exact test which showed that there was a greater proportion of patients who benefited from the stimulations (very much improved, much improved or minimally improved) in the non-opioid group than in the opioid group $(\mathrm{p}<.05)$. There was no difference between the two groups for the CT stimulations $(\mathrm{p}=.48)$.

\section{Influence of participants' characteristics on TENS analgesia}

In order to verify if participants' characteristics influenced TENS analgesia, we performed exploratory analyses to determine if there was an association between AT and CT analgesia (measured during TENS stimulation) and the variables “Age”, "Sex", "Time since onset of symptoms", "Pain rating index", "Physical functioning", "Depression scores" and "Pain catastrophizing". For both the opioid and non-opioid group, Spearman tests showed that none of these variables were correlated with AT or CT analgesia (all p-values $>.07$ ). For the opioid group, we also verified if there was an association between TENS (CT and AT) analgesia and the duration of opioid treatment. Again, the Spearman test revealed no significant correlation (all p-values $>.48$ ). 


\section{Linear regression model}

Because analyses of the participants' characteristics revealed that both groups were not fully comparable (see Table 2), we performed linear regression analyses to determine if the variable "Group" could still explain AT analgesia after adjusting for "Age", "Sex", "Time since onset of symptoms", "Pain rating index", "Physical functioning", "Depression scores" and "Pain catastrophizing". Because the dependant variable was not normally distributed, distributions of the residuals were inspected and analysed to confirm the validity of the regression model. Results showed that the variable "Group" was still significant to explain AT analgesia $(\beta=.65, p<.05)$ after the inclusion of the other variables in the regression analysis model. Except for the variable "Group", no variable was a significant predictor of AT analgesia (all p-values $>0.3$ ). Visual inspection and analyses of the standardized and unstandardized residuals using the Shapiro-Wilk test revealed that the residuals were normally distributed $(\mathrm{p}=.97)$.

\section{Discussion}

The current results suggest that the analgesic effect of AT is reduced in patients taking opioids on a regular basis. Conversely, the analgesic effect of CT appears to be preserved in these patients. Such observations are coherent with the work of Sluka et al. ${ }^{39}$ who showed that low frequency (but not high frequency) TENS was less effective in reducing secondary heat hyperalgesia in morphine-tolerant rats. They also confirm and extend the results of Solomon et al. ${ }^{41}$ who documented that the analgesic effect of TENS for post-operative pain was reduced in patients who had used narcotics prior to surgery compared to patients who had not used narcotics before surgery. 
The reduced analgesic effect of AT in opioid-treated patients was observed both for pain intensity and pain unpleasantness. Similarly, and for the other conditions where TENS was found to be effective (i.e. AT in participants from the non-opioid group and CT in participants from both groups), pain intensity and unpleasantness behaved similarly, with patients showing a reduction both in pain intensity and unpleasantness during and after TENS. Although closely linked, pain intensity (sensory-discriminative) and unpleasantness (motivational-affective) are two separate components of pain which are associated with distinct anatomical regions of the pain matrix. ${ }^{2}$ Our results indicate (i) that TENS application can reduce both the sensory-discriminative and motivationalaffective component of pain and (ii) that opioid consumption blocks the effect of AT on these two components concurrently.

Recent evidence from animal models suggests that TENS act on different opioid receptors, depending on the frequency employed. Specifically, low frequency TENS has been shown to activate $\mu$ opioid receptors whereas high frequency TENS has been shown to activate $\delta$ opioid receptors. ${ }^{20,38}$ These results are supported by human studies that showed that small doses of naloxone (blocking for the most part $\mu$ opioid receptors) ${ }^{17}$ are able to reverse AT but not CT analgesia. ${ }^{1,8,15,19,24,37,44}$ Alternately, large doses of naloxone (which are believed to ensure adequate blockage of all opioid receptor subtypes) ${ }^{3,5}$ were shown to reverse $\mathrm{CT}$ analgesia. ${ }^{23}$ The interaction between AT and opioids (but not between CT and opioids) observed in the present study indirectly confirm these observations. In particular, the reduced analgesic effect of AT in opioidtreated patients could be seen as a confirmation that AT and opioids are mediated through the same receptors (i.e. $\mu$ opioid receptors). Since cross-tolerance does not occur between 
$\mu$ and $\delta$ opioid receptors, ${ }^{21}$ the preserved analgesic effect of CT in these patients would further suggest that $\mathrm{CT}$ analgesia is mediated by non- $\mu$ opioid receptors (i.e. $\delta$ or $\kappa$ opioid receptors).

From a clinical perspective, our results suggest that a patient taking opioids on a regular basis would be less susceptible to benefit from AT stimulations. This does not exclude that some of these patients could benefit from AT stimulations. In fact, five out of 11 participants from the opioid group reported some improvements on the PGIC scale after AT stimulations, indicating that the analgesic effect of AT is not completely and systematically hindered in all patients taking opioids. However, and given the general pattern observed, clinicians would certainly be advised to initially try CT in this group of patients. This appears particularly important when we consider that previous exposition to ineffective treatments can significantly reduce the analgesic effect of subsequent treatments. $^{9}$

Recently, Ram et al. ${ }^{33}$ showed that the analgesic effect of a standard counterirritation paradigm (i.e. immersion of right hand in painfully cold water) was reduced in opioid- treated patients. The results from the present study support and extend the results of Ram et al. by showing that the analgesic efficacy of AT (another counter-irritation technique) is also reduced in opioid-treated patients. Taken together, these observations would suggest that the analgesic effect of counter-irritation techniques, in general, is reduced in opioid- treated patients. 


\section{Influence of participants' characteristics on TENS analgesia}

Our results showed that there was no association in both the opioid and nonopioid group between participants' characteristics and the analgesic effect of TENS. However, it is important to remember that the objective of the present study was not to evaluate the influence of these factors on TENS analgesia. Additional research looking at the effect of variables such as depression scores and pain catastrophizing level on the analgesic effect of TENS is warranted.

\section{Stimulation thresholds and intensities}

Our analyses revealed that there were no group differences for AT stimulation threshold and stimulation intensities. For CT however, stimulation threshold and intensities were consistently higher in the opioid group when compared to the non-opioid group. Traditionally, administration of opioids have been shown to induce hypoalgesia and thermal hypoaesthesia without alteration of fine touch and proprioception. ${ }^{4,16,26}$ Somewhat challenging this view are the observations of Park et al. ${ }^{31}$ who showed that the non-noxious perceptual thresholds to high frequency electrical stimulations (250 and $2000 \mathrm{~Hz}$ ) were decreased following alfentanil administration. On the contrary, there was no change in non-noxious perceptual thresholds when thresholds were measured with low frequency electrical stimulations $(5 \mathrm{~Hz})$ and conventional Von Frey hairs.

Physiologically, $\mu$ opioid receptors are present in small-sized and medium-sized sensory neurons. ${ }^{45}$ The elevation of warm and noxious detection thresholds following opioid administration is coherent with the function of these fibers which are known to transmit information relative to pain and temperature. ${ }^{25}$ The changes in non-noxious 
electrical thresholds are somewhat more puzzling when we consider that large-sized fibers do not typically express opioid receptors. ${ }^{35}$ The recent observations of Sasaki et al. ${ }^{35}$ could help to shed some light on these contradictory findings. In their study, Sasaki et al. showed that $\mu$ opioid receptors - which were present almost exclusively in smallsized and medium-sized sensory neurons in a group of naive mice - were largely expressed in large sensory neurons in mice suffering from herpetic pain.

Taken together, the results of Sasaki et al. and our results suggest that opioids can alter non-noxious electrical stimulation thresholds and that these alterations are particularly susceptible to occur in people suffering from certain pain conditions. It nevertheless remains unclear why we observed higher stimulation thresholds and intensities for non-noxious (high frequency) but not for noxious (low frequency)

stimulations. As suggested by Park et al. ${ }^{31}$, it is probable that the effect of opioids on the perception of electrical stimulation is highly dependant upon the frequency of the electrical stimulation. Clearly, future studies are necessary to examine this question.

\section{Limitations}

An important limitation of the present study concerns the group differences observed in participants' characteristics. For example, we observed higher Beck Depression Inventory scores in the opioid group compared to the non-opioid group, suggesting that participants from the opioid group had more symptoms of depression and more emotional distress. Although non-significant, a number of other measures also tended to be more elevated in the opioid group (e.g. McGill Pain Questionnaire scores). This raises some important questions. For instance, one could wonder if the reduced 
analgesic effect of AT in participants from the opioid group could not be explain by the fact that these patients had more symptoms of depression rather than because they took opioids on a regular basis. Without completely excluding this possibility, the preserved analgesic effect of CT in the opioid group tends to discredit this interpretation.

Obviously, and if the group differences were to be responsible for the difference in TENS analgesia, these differences would tend to be observed not only for AT but also for CT.

Nevertheless, and since CT and AT are believed to exert their analgesic effect via distinct neurophysiological mechanisms (i.e. segmental inhibition $v s$ descending inhibition), the preserved analgesic effect of CT cannot entirely rule out the possibility that the results for AT were not influenced by participants' characteristics. The group differences for the indices of depression appear particularly problematic when we consider that serotonin neurotransmission (which was shown to be altered in major depressive dirsorders $)^{6,28}$ plays a crucial role in descending pain modulation. ${ }^{29}$ In an attempt to dissipate these doubts, we performed a linear regression analysis to determine if the variable "Group" could still explain AT analgesia after adjusting for participants' characteristics. Results showed that the variable "Group" was still a significant predictor of AT analgesia despite the inclusion of the other variables in the regression analysis model, suggesting that the group differences in participants' characteristics are not responsible for the group difference in AT analgesia.

An additional limitation concerns the absence of placebo. By comparing the placebo condition to the active treatment condition, it would have been possible to determine if the analgesic effect of an intervention was superior to its placebo effect. Because the aim of the present study was to determine if the overall analgesic effect of 
TENS differed between opioid treated patients and opioid-naive patients (and not to determine if the analgesic effect of TENS was superior to placebo TENS), we did not include a placebo TENS condition.

Finally, it should be noted that the present study did not allow us to determine if the patients in the opioid group actually developed opioid tolerance. Thus, and despite the fact that these participants took opioids on a regular basis for more than 4 months, it is impossible to ascertain if the reduced analgesic effect of TA in this group of participants is attributable to a "cross-tolerance" effect. Questioning this cross-tolerance hypothesis is the work from Le Bars et al. ${ }^{22}$ who showed that a single intravenous injection of morphine could block counter-irritation analgesia in healthy participants, indicating that the antagonistic effect of opioids on counter-irritation analgesia is present well before the development of opioid tolerance. Conversely, the reduced analgesic of AT reported by Sluka et al. ${ }^{39}$ was observed on Sprague-Dawley rats who received morphine pellets for 10 days (a procedure which was shown by Sluka et al. to induce morphine tolerance). Whether the reduced analgesic of AT in our patients taking opioids is dependent on the induction of opioid tolerance remains unknown.

\section{Conclusion}

In the present study, we showed that the analgesic effect of CT was comparable between the opioid and non-opioid groups, suggesting preserved CT analgesia in opioidtreated patients. For AT however, the analgesic effect of TENS was only observed in the non-opioid group, with opioid-treated patients showing no change in pain scores during and after TENS stimulation. Taken together, these results propose that AT is less 
effective than $\mathrm{CT}$ in opioid-treated patients and that $\mathrm{CT}$ stimulations should be preferred in patients taking opioids on a regular basis.

\section{Acknowledgments}

The authors wish to thank Joël Charlebois for his thoughtful comments on the manuscript and Marie-Ève Mauger for her help with data collection. Part of this work served as a partial fulfillment of Ph.D. degree by Guillaume Léonard. Guillaume Léonard received postgraduate scholarships from FRSQ (Fonds de recherche en santé du Québec) and IRSST (Institut de recherche en santé et sécurité au travail). Serge Marchand is supported by CIHR (Canada) and FRSQ (Québec) and is a member of the Centre de recherche clinique Étienne-Le-Bel (CRCELB) of the CHUS. 


\section{References}

(1) Abram SE, Reynolds AC, Cusick JF: Failure of naloxone to reverse analgesia from transcutaneous electrical stimulation in patients with chronic pain. Anesth Analg 60:81-84, 1981.

(2) Apkarian AV, Bushnell MC, Treede RD, Zubieta JK: Human brain mechanisms of pain perception and regulation in health and disease. Eur J Pain 9:463-484, 2005.

(3) Benedetti F: The opposite effects of the opiate antagonist naloxone and the cholecystokinin antagonist proglumide on placebo analgesia. Pain 64:535-543, 1996.

(4) Brennum J, rendt-Nielsen L, Horn A, Secher NH, Jensen TS: Quantitative sensory examination during epidural anaesthesia and analgesia in man: effects of morphine. Pain 52:75-83, 1993.

(5) Bruehl S, Chung OY, Ward P, Johnson B, McCubbin JA: The relationship between resting blood pressure and acute pain sensitivity in healthy normotensives and chronic back pain sufferers: the effects of opioid blockade. Pain 100:191-201, 2002.

(6) Celada P, Puig M, margos-Bosch M, Adell A, Artigas F: The therapeutic role of 5-HT1A and 5-HT2A receptors in depression. J Psychiatry Neurosci 29:252-265, 2004.

(7) Chandran P, Sluka KA: Development of opioid tolerance with repeated transcutaneous electrical nerve stimulation administration. Pain 102:195-201, 2003.

(8) Chapman CR, Benedetti C: Analgesia following transcutaneous electrical stimulation and its partial reversal by a narcotic antagonist. Life Sci 21:16451648, 1977.

(9) Charron J, Rainville P, Marchand S: Direct comparison of placebo effects on clinical and experimental pain. Clin J Pain 22:204-211, 2006.

(10) Chen CC, Johnson MI: An investigation into the hypoalgesic effects of high- and low-frequency transcutaneous electrical nerve stimulation (TENS) on experimentally-induced blunt pressure pain in healthy human participants. J Pain 11:53-61, 2010.

(11) Dworkin RH, Turk DC, Farrar JT, Haythornthwaite JA, Jensen MP, Katz NP, Kerns RD, Stucki G, Allen RR, Bellamy N, Carr DB, Chandler J, Cowan P, Dionne R, Galer BS, Hertz S, Jadad AR, Kramer LD, Manning DC, Martin S, McCormick CG, McDermott MP, McGrath P, Quessy S, Rappaport BA, Robbins 
W, Robinson JP, Rothman M, Royal MA, Simon L, Stauffer JW, Stein W, Tollett J, Wernicke J, Witter J: Core outcome measures for chronic pain clinical trials: IMMPACT recommendations. Pain 113:9-19, 2005.

(12) Facchinetti F, Sandrini G, Petraglia F, Alfonsi E, Nappi G, Genazzani AR: Concomitant increase in nociceptive flexion reflex threshold and plasma opioids following transcutaneous nerve stimulation. Pain 19:295-303, 1984.

(13) Fishbain DA, Chabal C, Abbott A, Heine LW, Cutler R: Transcutaneous electrical nerve stimulation (TENS) treatment outcome in long-term users. Clin J Pain 12:201-214, 1996.

(14) Foley RA: Transcutaneous electrical nerve stimulation. In: Manual for Physical Agents, 5th edition (Hayes K. W., Ed), Prentice Hall Health, New Jersey, 2000, pp. 121-147.

(15) Freeman TB, Campbell JN, Long DM: Naloxone does not affect pain relief induced by electrical stimulation in man. Pain 17:189-195, 1983.

(16) Glynn CJ, Mather LE, Cousins MJ, Graham JR, Wilson PR: Peridural meperidine in humans: analgesic response, pharmacokinetics, and transmission into CSF. Anesthesiology 55:520-526, 1981.

(17) Gourlay GK: Advances in opioid pharmacology. Support Care Cancer 13:153$159,2005$.

(18) Guy W, ECDEU assessment manual for psychopharmacology (DHEW Publication No. ADM 76-338), Government Printing Office, Washington DC, 1976.

(19) Hansson P, Ekblom A, Thomsson M, Fjellner B: Influence of naloxone on relief of acute oro-facial pain by transcutaneous electrical nerve stimulation (TENS) or vibration. Pain 24:323-329, 1986.

(20) Kalra A, Urban MO, Sluka KA: Blockade of opioid receptors in rostral ventral medulla prevents antihyperalgesia produced by transcutaneous electrical nerve stimulation (TENS). J Pharmacol Exp Ther 298:257-263, 2001.

(21) Kalso EA, Sullivan AF, McQuay HJ, Dickenson AH, Roques BP: Cross-tolerance between mu opioid and alpha-2 adrenergic receptors, but not between mu and delta opioid receptors in the spinal cord of the rat. J Pharmacol Exp Ther 265:551$558,1993$.

(22) Le Bars D, Willer JC, De BT: Morphine blocks descending pain inhibitory controls in humans. Pain 48:13-20, 1992. 
(23) Leonard G, Rodrigue M, Cloutier C, Marchand S: Deciphering the role of endogenous opioids in high frequency TENS using high and low doses of naloxone. IASP 12th World Congress on Pain (Glasgow), 2008.

(24) Lundeberg T, Bondesson L, Lundstrom V: Relief of primary dysmenorrhea by transcutaneous electrical nerve stimulation. Acta Obstet Gynecol Scand 64:491497, 1985.

(25) Lundy-Ekman L: Somatosensory system. In: Neuroscience : Fundamentals for Rehabilitation, W.B. Saunders Company, Philadelphia, 1998, pp. 85-105.

(26) Magora F, Olshwang D, Eimerl D, Shorr J, Katzenelson R, Cotev S, Davidson JT: Observations on extradural morphine analgesia in various pain conditions. $\mathrm{Br} \mathrm{J}$ Anaesth 52:247-252, 1980.

(27) Melzack R, Wall PD: Pain mechanisms: a new theory. Science 150:971-979, 1965.

(28) Meyer JH: Imaging the serotonin transporter during major depressive disorder and antidepressant treatment. J Psychiatry Neurosci 32:86-102, 2007.

(29) Millan MJ: Descending control of pain. Prog Neurobiol 66:355-474, 2002.

(30) O'Sullivan SB, Schmitz TJ, Physical Rehabilitation: Assessment and Treatment, F. A. Davis Company, Philadelphia, 2000.

(31) Park R, Wallace MS, Schulteis G: Relative sensitivity to alfentanil and reliability of current perception threshold vs von Frey tactile stimulation and thermal sensory testing. J Peripher Nerv Syst 6:232-240, 2001.

(32) Price DD, McGrath PA, Rafii A, Buckingham B: The validation of visual analogue scales as ratio scale measures for chronic and experimental pain. Pain 17:45-56, 1983.

(33) Ram KC, Eisenberg E, Haddad M, Pud D: Oral opioid use alters DNIC but not cold pain perception in patients with chronic pain - new perspective of opioidinduced hyperalgesia. Pain 139:431-438, 2008.

(34) Robinson AJ, Snyder-Mackler L, Clinical Electrophysiology: Electrotherapy and Electrophysiologic Testing, 3rdth edition, Lippincott Williams \& Wilkins, Philadelphia, 2007.

(35) Sasaki A, Nakashima Y, Takasaki I, Andoh T, Shiraki K, Kuraishi Y: Morphine inhibits herpetic allodynia through mu-opioid receptors induced in Abeta-fiber neurons. Neuroreport 19:975-979, 2008. 
(36) Schug S, Gandham N: Opioids: clinical use. In: Wall and Melzack's Textbook of Pain, 5th edition (McMahon S. B. and Koltzenburg M., Eds), Elsevier Churchill Livingstone, China, 2006, pp. 443-457.

(37) Sjolund B, Terenius L, Eriksson M: Increased cerebrospinal fluid levels of endorphins after electro-acupuncture. Acta Physiol Scand 100:382-384, 1977.

(38) Sluka KA, Deacon M, Stibal A, Strissel S, Terpstra A: Spinal blockade of opioid receptors prevents the analgesia produced by TENS in arthritic rats. J Pharmacol Exp Ther 289:840-846, 1999.

(39) Sluka KA, Judge MA, McColley MM, Reveiz PM, Taylor BM: Low frequency TENS is less effective than high frequency TENS at reducing inflammationinduced hyperalgesia in morphine-tolerant rats. Eur J Pain 4:185-193, 2000.

(40) Sluka KA, Walsh D: Transcutaneous electrical nerve stimulation: basic science mechanisms and clinical effectiveness. J Pain 4:109-121, 2003.

(41) Solomon RA, Viernstein MC, Long DM: Reduction of postoperative pain and narcotic use by transcutaneous electrical nerve stimulation. Surgery 87:142-146, 1980.

(42) Warke K, Al-Smadi J, Baxter D, Walsh DM, Lowe-Strong AS: Efficacy of transcutaneous electrical nerve stimulation (tens) for chronic low-back pain in a multiple sclerosis population: a randomized, placebo-controlled clinical trial. Clin J Pain 22:812-819, 2006.

(43) Willer JC, Bouhassira D, Le BD: [Neurophysiological bases of the counterirritation phenomenon:diffuse control inhibitors induced by nociceptive stimulation]. Neurophysiol Clin 29:379-400, 1999.

(44) Willer JC, Roby A, Boulu P, Boureau F: Comparative effects of electroacupuncture and transcutaneous nerve stimulation on the human blink reflex. Pain 14:267-278, 1982.

(45) Yamamoto J, Kawamata T, Niiyama Y, Omote K, Namiki A: Down-regulation of mu opioid receptor expression within distinct subpopulations of dorsal root ganglion neurons in a murine model of bone cancer pain. Neuroscience 151:843853,2008 . 


\section{Figure Legends}

Figure 1. Pain intensity before, during and after TENS for (A) CT and (B) AT. Both groups (opioid and non-opioid) showed a reduction in pain intensity during and after CT (all p-values $<.01$ ) when compared to baseline. For AT, there was a significant reduction in pain intensity during and after TENS for the non-opioid group (all p-values <.01) but not for the opioid group $(\mathrm{p}=.09)$. Between-group comparisons revealed that there were significant differences between the opioid and non-opioid group during $(\mathrm{p}<.01)$ and after $(\mathrm{p}<.05)$ AT.

A

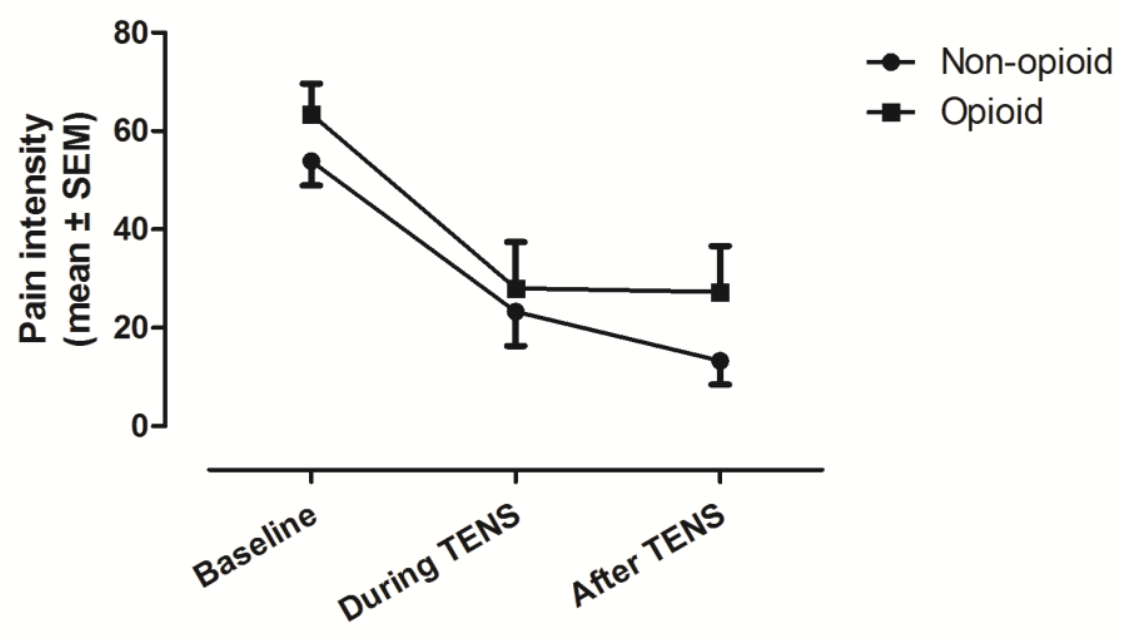

B
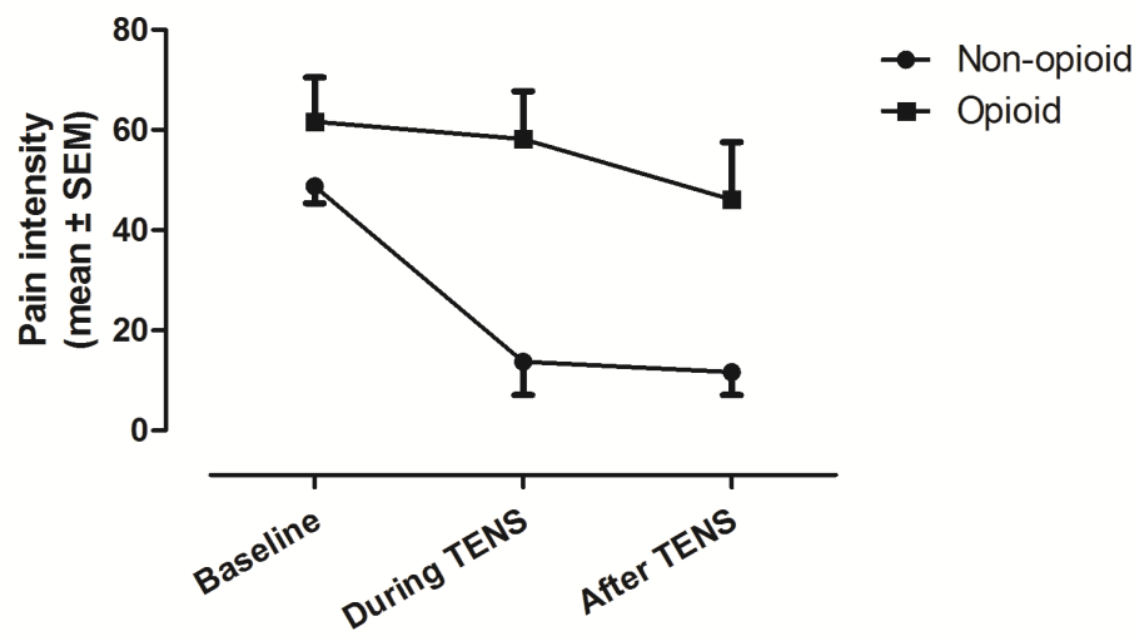
Figure 2. Pain unpleasantness before, during and after TENS for (A) CT and (B) AT. There was a significant reduction in pain unpleasantness during and after CT both in the opioid $(\mathrm{p}<.05)$ and non-opioid group $(\mathrm{p}<.01)$ when compared to baseline. For AT, there was a significant reduction in pain unpleasantness during and after TENS for the nonopioid group $(\mathrm{p}<.01)$ but not for the opioid group $(\mathrm{p}=.36)$. Between-group comparisons revealed that there were significant differences between the opioid and non-opioid group during $(\mathrm{p}<.01)$ and after $(\mathrm{p}<.05)$ AT.

A

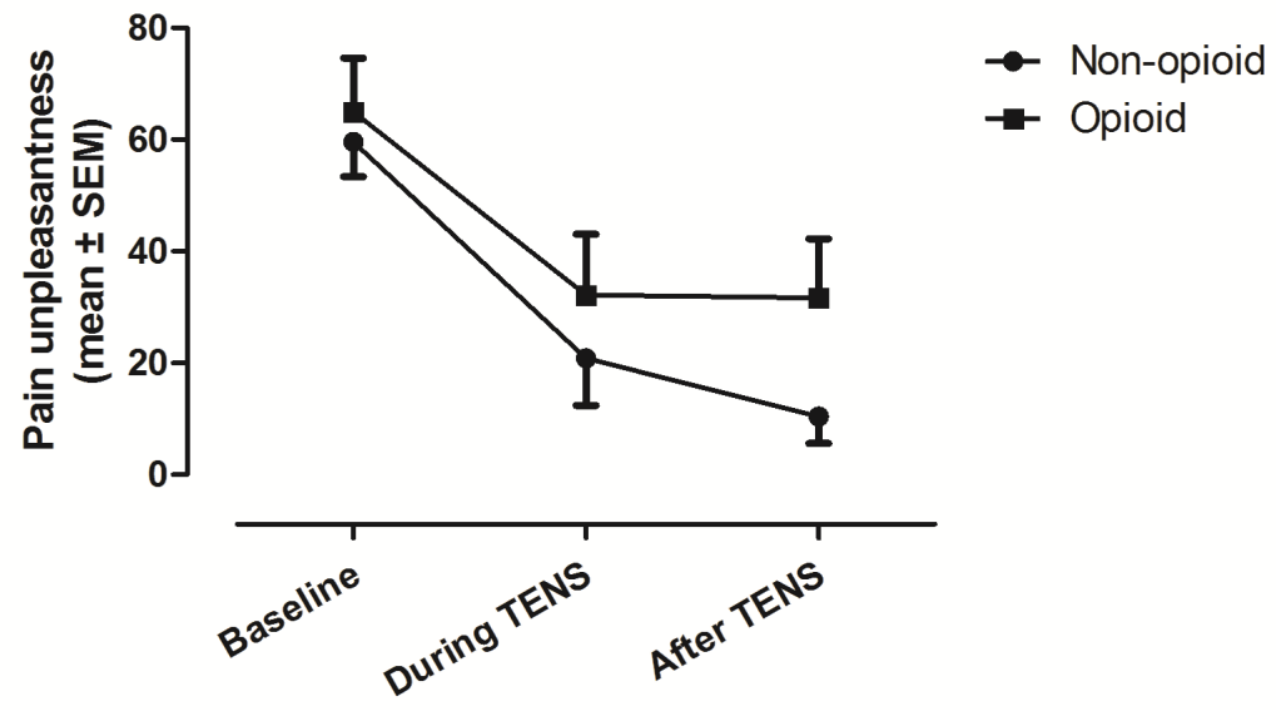

B

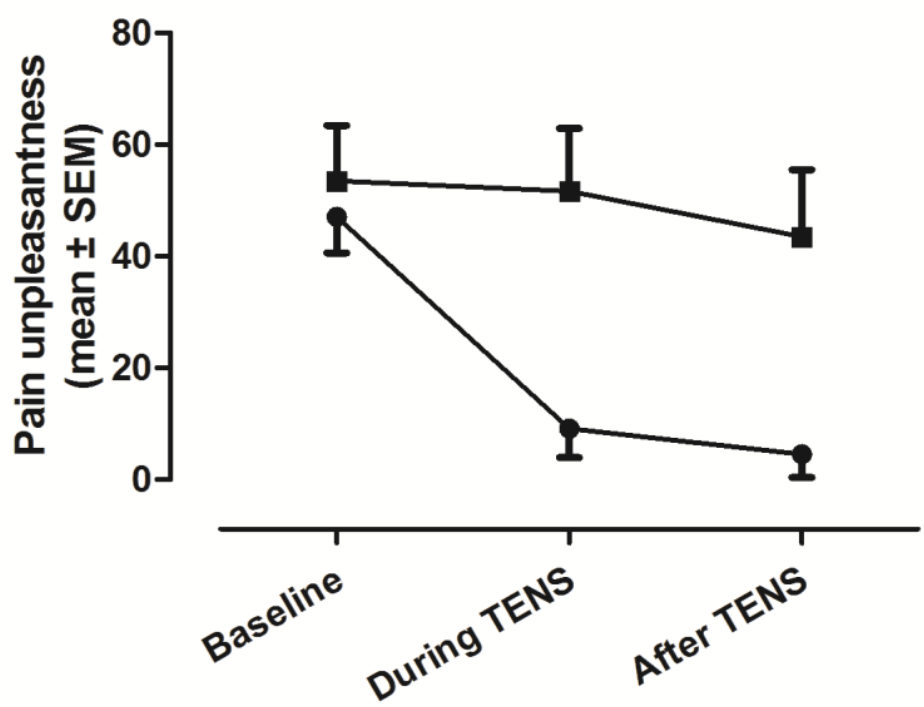


Figure 3. TENS analgesia during CT and AT stimulations $(* * \mathrm{p}<.01)$.

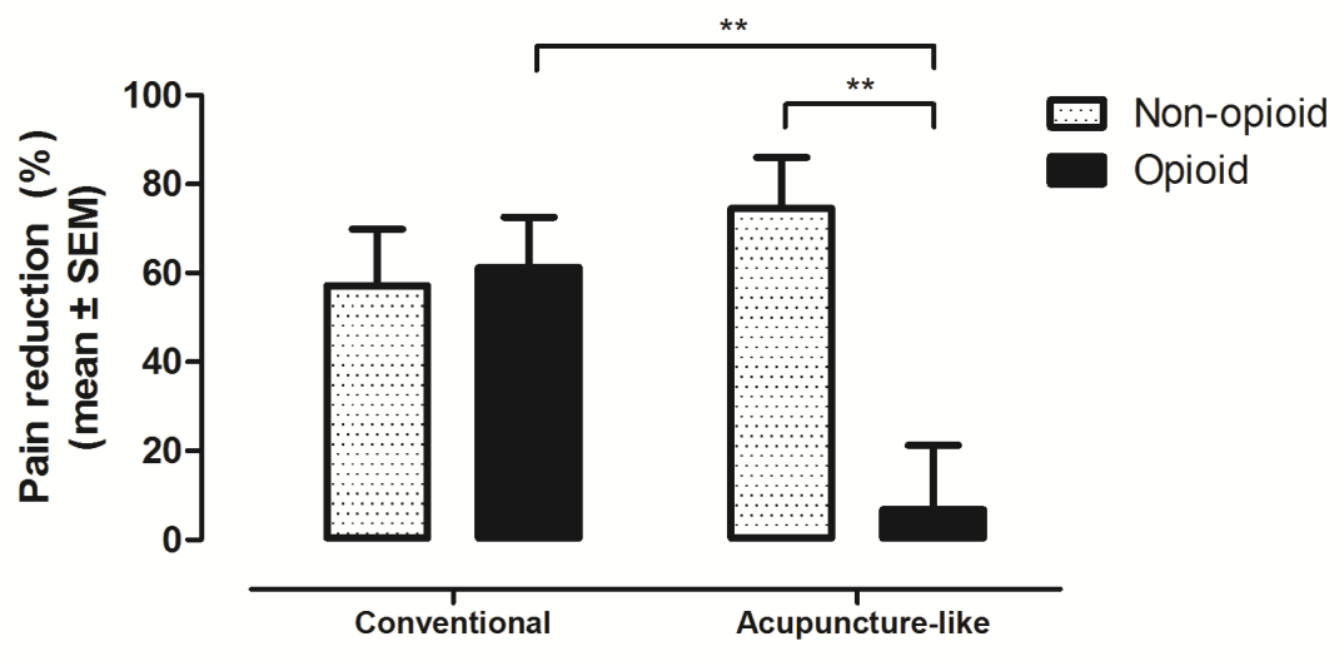

